\title{
Increasing the number of masticatory cycles is associated with reduced appetite and altered postprandial plasma concentrations of gut hormones, insulin and glucose
}

\author{
Yong Zhu ${ }^{1}$, Walter H. Hsu ${ }^{2}$ and James H. Hollis ${ }^{1 *}$ \\ ${ }^{1}$ Department of Food Science and Human Nutrition, Iowa State University, Ames, IA 50011, USA \\ ${ }^{2}$ Department of Biomedical Sciences, Iowa State University, Ames, IA 50011, USA \\ (Submitted 14 June 2012 - Final revision received 17 September 2012 - Accepted 12 October 2012 - First published online 27 November 2012)
}

\section{Abstract}

To determine the influence of masticatory efficiency on postprandial satiety and glycaemic response, twenty-one healthy males were recruited for this randomised cross-over trial. The participants consumed a fixed amount of pizza provided in equal-sized portions by chewing each portion either fifteen or forty times before swallowing. Subjective appetite was measured by appetite questionnaires at regular intervals for $3 \mathrm{~h}$ after the meal and plasma samples were collected for the measurement of selected satiety-related hormones, glucose, insulin and glucose-dependent insulinotropic peptide (GIP) concentrations. An ad libitum meal was provided shortly after the last blood sample was drawn and the amount eaten recorded. Compared with fifteen chews, chewing forty times per portion resulted in lower hunger $(P=0.009)$, preoccupation with food $(P=0.005)$ and desire to eat $(P=0.002)$. Meanwhile, plasma concentrations of glucose $(P=0.024)$, insulin $(P<0.001)$ and GIP $(P<0.001)$ were higher following the forty-chews meal. Chewing forty times before swallowing also resulted in a higher plasma cholecystokinin concentration $(P=0.045)$ and a trend towards a lower ghrelin concentration $(P=0.051)$. However, food intake at the subsequent test meal did not differ $(P=0.851)$. The results suggest that a higher number of masticatory cycles before swallowing may provide beneficial effects on satiety and facilitate glucose absorption.

\section{Key words: Mastication: Satiety: Glycaemic response}

Due to the high prevalence of overweight and obesity, new strategies to aid weight management are required. This would be helped by a better understanding of the factors that influence satiety so that this information can be used to identify individuals at increased risk of weight gain or for the development of improved therapeutic diets ${ }^{(1,2)}$. It has been reported that a fast eating rate, a larger bite size or shorter oral processing time can promote overeating ${ }^{(3-8)}$ and is associated with elevated body weight or risk of weight gain ${ }^{(9-11)}$. A key influence on eating rate is masticatory efficiency (i.e. the number of masticatory cycles required before swallowing); however, its influence on satiety has gained little attention.

The primary purpose of mastication is to reduce the particle size of a food to form a bolus for swallowing. There is substantial inter-individual variation in the number of masticatory cycles required to form a bolus, and it has been reported that the number of masticatory cycles made before swallowing ranges between 9 and 65 for carrots and 14 and 44 for Brazil nuts ${ }^{(12)}$. Moreover, food preparation methods, such as chopping, roasting or salting, also influence the number of masticatory cycles required before swallowing ${ }^{(13)}$. These differences in masticatory efficiency could influence satiety through several mechanisms. First, studies using rodents report that mastication has a direct effect on satiety through histaminergic activation of the ventromedial hypothalamus and paraventricular nucleus ${ }^{(14,15)}$. Second, mastication is a key stimulus of cephalic phase responses $(\mathrm{CPR})^{(16)}$, and increasing masticatory effort before swallowing may increase the CPR of hormones related to appetite such insulin, cholecystokinin (CCK) and pancreatic polypeptide ${ }^{(17-19)}$. Third, increasing the number of masticatory cycles would increase oral processing time, and recent studies have shown that increasing oral processing time reduces appetite or food intake $^{(6,20)}$. It will also slow down eating rate, which has been associated with increased satiety by one study ${ }^{(21)}$, although these results were not confirmed by another study $^{(22)}$. Taken together, there are good reasons to believe that increasing the number of masticatory cycles before swallowing will increase satiety.

Abbreviations: CCK, cholecystokinin; CPR, cephalic phase responses; GIP, glucose-dependent insulinotropic peptide. 
Recent studies report that making a higher number of masticatory cycles before swallowing increases satiety ${ }^{(23,24)}$. Cassady et al. ${ }^{(23)}$ found that chewing almonds forty times before swallowing reduces appetite and modulates plasma concentrations of several hormones compared with chewing fifteen times. However, almonds contain a relatively high amount of lipids, which are a key stimulus for several putative satiety hormones ${ }^{(25)}$, and as mastication increased the release of lipids from the food matrix, it may be that increased bioaccessibility of lipids was the primary reason for enhanced satiety rather than mastication. A study conducted by Li et al. ${ }^{(24)}$ showed that increasing the number of chewing cycles from fifteen to forty when eating pork pie increased satiety in Chinese adults, which was potentially due, in part, to differences in postprandial secretion of gut-derived hormones related to satiety. Further studies are warranted to determine if differences in the number of masticatory cycles made before swallowing influence appetite using different test foods or other population groups.

Based on previous studies, we hypothesised that a higher number of masticatory cycles before swallowing will increase satiety. This effect will be modulated through changes in plasma concentrations of gut-derived hormones that are related to appetite.

\section{Subjects and methods}

\section{Subjects}

The present study was advertised by a mass e-mail sent to Iowa State University students and staff and by fliers distributed throughout the local community. Individuals interested in the study were invited to attend a screening session to determine their eligibility for the study. During this session, the participant's height was measured by a stadiometer and weight was measured using calibrated weighing scales, with the participant dressed in a paper gown. The participant was required to void the bladder before this measurement. Inclusion criteria were: male, aged $18-40$ years, BMI $20 \cdot 0-29 \cdot 9 \mathrm{~kg} / \mathrm{m}^{2}$, full set of natural teeth and a willingness to eat the test foods. Potential participants were excluded from the study if they: had presence or history of gastrointestinal disease, had presence of other chronic or acute diseases, currently using medication that affects appetite, were restrained eaters $(>13$ on the restraint section of the Three-Factor Eating Questionnaire ${ }^{(26)}$ ) and had allergy or intolerance to the test foods. Participants were instructed to taste and rate the palatability of the test foods using a nine-point scale. Any participants with a score below 5 were excluded. Participants were informed that the purpose of the study was to investigate the effect of chewing on plasma nutrients. When the participants completed the study, they were informed about the true purpose of the study and given the option to withdraw their data from the study. The present study was conducted according to the guidelines laid down in the Declaration of Helsinki, and all procedures involving human subjects were approved by the Iowa State University Institutional Review Board. Written informed consent was obtained from all the subjects.

\section{Test meals}

Freschetta brick oven fire baked 5-cheese pizza (Schwan Food Company) was used as the test food. Nutrient labelling by the manufacturer reported that each pizza provided $51 \mathrm{~g}$ carbohydrate, $23 \mathrm{~g}$ fat, $22 \mathrm{~g}$ protein and $2050 \mathrm{~kJ}$ ( $490 \mathrm{kcal}$ ) energy with a total weight of $183 \mathrm{~g}$. Each participant consumed one complete pizza during each test session. The pizza was baked at $204^{\circ} \mathrm{C}\left(400^{\circ} \mathrm{F}\right)$ in a conventional oven for $15 \mathrm{~min}$ and allowed to cool to a comfortable eating temperature before serving.

An ad libitum pasta meal was served $3 \mathrm{~h}$ after eating the pizza meal. Meals were provided in $3766 \mathrm{~kJ}$ (900 kcal) portions, made with $150 \mathrm{~g}$ Barilla spaghetti (Barilla America, Inc.), $375 \mathrm{~g}$ Barilla Marinara sauce with imported olive oil (Barilla America, Inc.), $37.5 \mathrm{~g}$ shredded parmesan cheese (Wal-Mart Stores, Inc.) and $5 \cdot 1 \mathrm{~g}$ salt (Wal-Mart Stores, Inc.). Meals were prepared using a standard procedure and mixed well before being served to the participants.

\section{General procedure}

A preliminary session was arranged for all eligible participants to determine a suitable pizza portion size (mouthful) for use in the study. Results from this preliminary session indicated a portion size of $3.8 \times 2.5 \mathrm{~cm}$ could be safely swallowed by all the participants after fifteen chewing cycles. For both test sessions, the pizza was cut into twenty-four portions of $3.8 \times 2.5 \mathrm{~cm}$.

Following the preliminary session, participants attended two test sessions that were separated by at least $7 \mathrm{~d}$. The treatment order was randomised. Participants were required to report to the laboratory at 07.30 hours after an overnight fast. The participants were instructed to refrain from drinking alcoholic beverages during $24 \mathrm{~h}$ before the test session, but no other restrictions were placed on their eating or drinking habits. They were also asked to refrain from strenuous physical activity for $24 \mathrm{~h}$ prior to the test session. An indwelling catheter was inserted into their non-dominant arm, and following a $30 \mathrm{~min}$ acclimatisation period, a baseline blood sample was drawn. The participants also completed an appetite questionnaire to determine their baseline subjective appetite. The questionnaire posed four questions: How hungry do you feel right now? How full do you feel right now? How preoccupied with food are you right now? What is your desire to eat right now? Responses were captured using a $100 \mathrm{~mm}$ visual analogue scale. The visual analogue scale was anchored with diametrically opposed statements in each end (e.g. not hungry at all, as hungry as I have ever felt). Participants were instructed to draw a vertical marker on the scale at the position they felt reflected their current strength of their appetitive feeling.

Immediately following the baseline measurements, the participants were presented with the pizza test meal. Depending on the test session, participants were instructed to chew each portion fifteen or forty times before swallowing the complete mouthful. A study investigator was present while the participant was eating to confirm that the participant followed the 
eating instructions. Meal duration was measured to the nearest $1 \mathrm{~min}$, and it was 8 (SEM 1) min for the fifteen chews session and 20 (SEM 1) $\mathrm{min}$ for the forty chews session.

Immediately after the participant finished the pizza meal, a fresh appetite questionnaire was completed and a blood sample was taken $\left(t_{0}\right)$. Further blood samples were collected and appetite questionnaires completed at $t_{0}+15,30,45,60$, 90, 120 and $180 \mathrm{~min}$. Throughout the test session, participants were required to remain seated in a quiet room, free from food cues, and were not allowed to consume other foods or drinks. The participants were allowed to read or use their computer during the test session. While other participants were also in the laboratory at the same time, they were isolated from each other by the use of screens. After the final blood draw, the catheter was removed and the participants were allowed to rest for $5 \mathrm{~min}$ before being presented with the pasta meal. Participants were instructed to eat until comfortably full and they were informed that they could request more of the pasta meal. No instruction regarding mastication was given. Each bowl of food was weighed before and after serving, without the subjects' knowledge, and the amount consumed was recorded.

\section{Hormones and glucose measurement}

Blood was drawn into $4 \mathrm{ml}$ EDTA-coated vacutainer tubes and mixed with $400 \mu \mathrm{l}$ of $10000 \mathrm{KIU}(1.4 \mathrm{mg}) / \mathrm{ml}$ aprotinin and then centrifuged at $3000 \mathrm{~g}$ at $4^{\circ} \mathrm{C}$ for $15 \mathrm{~min}$. The plasma was then divided into aliquots and stored at $-80^{\circ} \mathrm{C}$ until analysis. Insulin was assayed by RIA, as described previously ${ }^{(27)}$. Human insulin was used as standard. The assay had a detection range of $0 \cdot 78-200 \mu \mathrm{U}(5 \cdot 42-1389 \mathrm{pmol}) / \mathrm{ml}$. The intraassay $\mathrm{CV}$ was $13 \%$ and the inter-assay $\mathrm{CV}$ was $8 \%$ at $20 \mu \mathrm{U}$ (139 pmol)/ml. Glucose-dependent insulinotropic peptide (GIP) was assayed by RIA using 1:5000 rabbit anti-human GIP antibody (Phoenix Pharmaceuticals); the assay had a detection range of $0 \cdot 1-6 \cdot 4 \mathrm{ng} / \mathrm{ml}$, with intra-assay $\mathrm{CV}$ of $7 \%$ and inter-assay CV of $12 \%$ at $0.5 \mathrm{ng} / \mathrm{ml}$. CCK was assayed by RIA using rabbit anti-CCK-8 antibody 92128 diluted in the ratio 1:800 (the antibody was a kind gift from Dr Jens Rehfeld, University of Copenhagen). The assay was able to measure CCK from 4 to $128 \mathrm{pg} / \mathrm{ml}$. The intra-assay CV was $8 \%$ and inter-assay CV was $15 \%$ at $50 \mathrm{pg} / \mathrm{ml}$. Ghrelin was analysed by RIA using antibody $\mathrm{T}-4745$ purchased from Bachem. The assay had a determination range from 0.05 to $12 \cdot 8 \mathrm{ng} / \mathrm{ml}$. The intra-assay CV was $10 \%$ and inter-assay CV was $7 \%$ at $0.5 \mathrm{ng} / \mathrm{ml} .{ }^{125} \mathrm{I}$-Tracers used for RIA were purchased from PerkinElmer. Plasma glucose was assayed using a biochemical analyser (YSI Life Sciences, Model 2700 select).

\section{Statistical analysis}

Power calculations suggested that a sample size of eighteen is required to detect a change of $10 \%$ in overall mean for subjective appetite, concentrations of biomarkers and food intake, at the power of 0.8 and the significance level of 0.05 . An $8-10 \%$ reduction in appetite is considered to be practically relevant ${ }^{(28)}$. In the present study, $15 \%$ more participants ( $n$ 21) were recruited in case of possible drop-out during the study. All data are presented as means with their standard error. Statistical Analysis Software (version 9.2, 2008, SAS Institute, Inc.) was used to perform the statistical analysis. Plasma hormone data were log-transformed before analysis, as they were not normally distributed. A mixed model of repeatedmeasures ANOVA (Proc Mixed, SAS; SAS Institute, Inc.) was used to test the overall treatment effect, time effect and treatment $\times$ time interaction on subjective appetite ratings and plasma parameters. Baseline values were included as covariates and subjects were added as a random variable in the model. There was no significant effect of BMI on any of the outcome measures, so the data from all participants were pooled. Post hoc analysis was performed by Bonferroni-adjusted pairwise comparison of responses from the same time point. Difference in food intake at the ad libitum meal was tested by a paired $t$ test.

\section{Results}

\section{Participant characteristics}

Participants ( $n$ 21) had a mean age of 24 (SEM 1) years (range $18-36$ years) with a BMI of 24.8 (SEM 0.6$) \mathrm{kg} / \mathrm{m}^{2}$ (range $20 \cdot 3-28 \cdot 3 \mathrm{~kg} / \mathrm{m}^{2}$ ).

\section{Subjective appetite}

Fig. 1 illustrates subjective appetite responses following the fifteen or forty chews treatment. A significant main effect of time was found for all parameters $(P<0 \cdot 05)$, but there were no statistically significant treatment $\times$ time interactions $(P>0.05)$.

There was a significant main effect of chewing on hunger $(F(1,299)=6.92, P=0.009)$, with hunger being lower following the forty chews condition. A significant main effect of chewing on preoccupation with food and desire to eat was also found, with both being lower following forty chews $(F(1,299)=8.17, \quad P=0.005$ and $F(1,299)=9.59, \quad P=0.002$, respectively). There was no main effect of chewing on fullness $(F(1,299)=0 \cdot 06, P=0 \cdot 813)$.

\section{Glucose- and appetite-related hormones}

Fig. 2 shows plasma concentrations of glucose, insulin, GIP, CCK and ghrelin following the different chewing conditions. A main effect of time was significant for all parameters $(P<0.05)$, except CCK $(P=0.073)$.

There was a significant main effect of chewing on plasma glucose $(F(1,299)=5 \cdot 19, P=0 \cdot 024)$. Post hoc analysis revealed that plasma glucose was significantly higher at 0 min when forty chews were made $(P<0 \cdot 001)$. A significant main effect of chewing on plasma insulin was also found $(F(1,299)=19 \cdot 55, P<0.001)$, with post hoc analysis revealing that insulin was significantly higher at 0 and $15 \mathrm{~min}$ ( $P<0.001$ and $P=0.017$, respectively) following the forty chews condition. There was a significant main effect of chewing on GIP $(F(1,299)=22 \cdot 81, P<0 \cdot 001)$. Post boc analysis revealed a higher GIP response at $0 \mathrm{~min}(P<0.001)$ and $15 \mathrm{~min} \quad(P<0 \cdot 001)$ following the forty chews condition. 

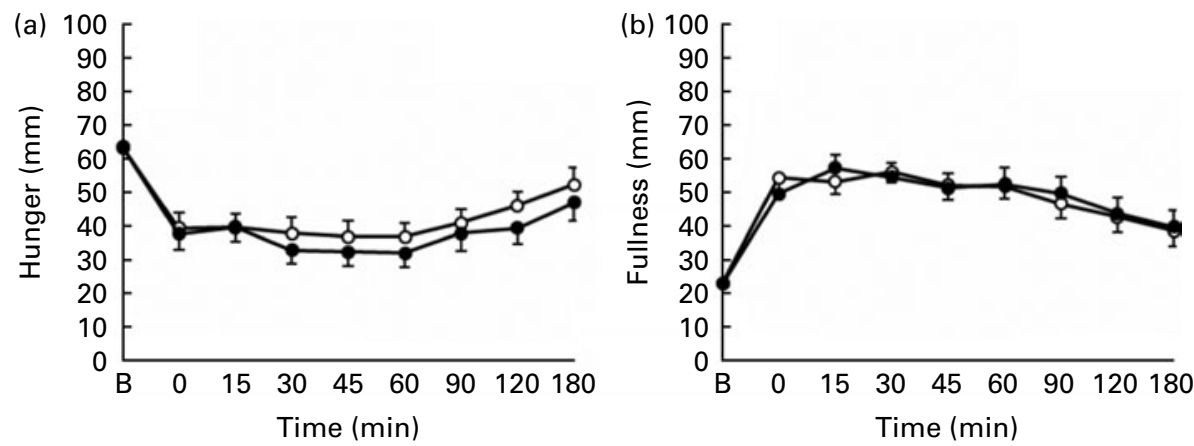

(c)

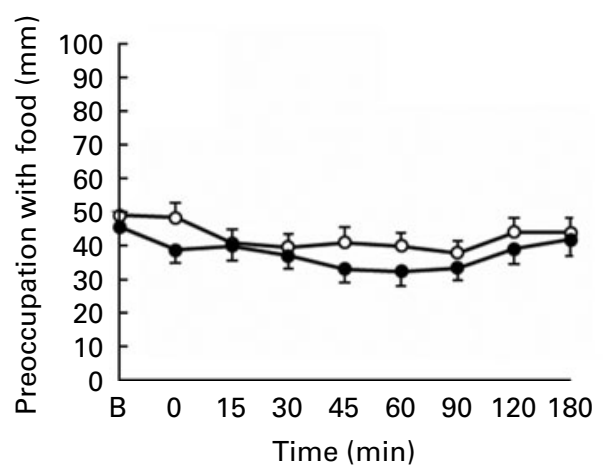

(d)

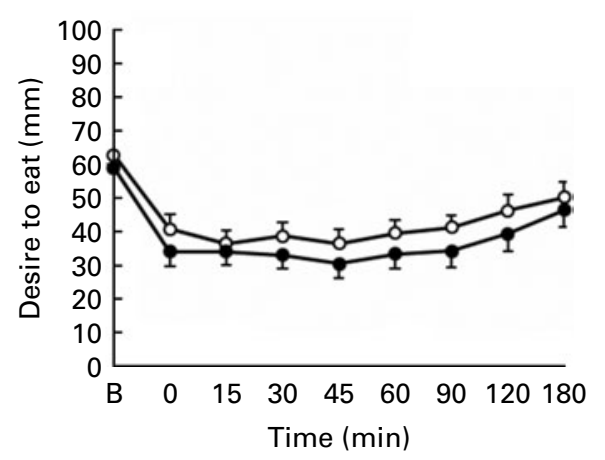

Fig. 1. (a) Hunger, (b) fullness, (c) preoccupation with food and (d) desire to eat following fifteen ( $\left.-\mathrm{O}_{-}\right)$and forty ( - - ) chews conditions. Values are means, with their standard errors represented by vertical bars ( $n$ 21). Mean values were significantly different for the main effect of treatment on hunger, preoccupation with food and desire to eat $(P=0.009, P=0.005$ and $P=0.002$, respectively). Mean value was not significantly different for the main effect of treatment on fullness $(P=0 \cdot 813)$.

A significant main effect of chewing on plasma CCK was found $(F(1,299)=4 \cdot 07, \quad P=0 \cdot 045)$. At $180 \mathrm{~min}$, CCK was significantly higher in the forty chews condition $(P=0.037)$. There was a trend towards a significant main effect of chewing on ghrelin $(F(1,299)=3 \cdot 83, P=0 \cdot 051)$, with lower ghrelin following the forty chews condition.

\section{Food intake}

There was no difference in food intake at the subsequent ad libitum meal after $3 \mathrm{~h}$ (forty chews: 417.4 (SEM 54.5) g $v$. fifteen chews: $410 \cdot 2$ (SEM 44.5) g; $P=0.851$ ).

\section{Discussion}

The present study found that increasing the number of chewing cycles before swallowing is associated with reduced postprandial hunger, preoccupation with food and desire to eat. This also resulted in a higher postprandial plasma concentration of CCK and reduced postprandial ghrelin. However, there was no difference in food intake at a meal served $3 \mathrm{~h}$ after the test meal. While the present study suggests that a higher number of masticatory cycles before swallowing was associated with reduced postprandial appetite, it was also associated with increased plasma concentrations of insulin and glucose.

Data from this present study are supportive of findings from previous studies that increasing the number of masticatory cycles before swallowing increases satiety, as measured by subjective appetite questionnaires ${ }^{(23,24)}$. These data raise the possibility that efficient eaters (i.e. individuals who use few masticatory cycles to form a bolus) may be at increased risk of weight gain due to reduced satiety. While epidemiological studies report that a fast eating rate is associated with a higher BMI or risk of weight gain ${ }^{(9-11)}$, it is not clear to what degree differences in masticatory efficiency contributed to eating rate. Further studies are required to determine if there is a difference in the satiety response between efficient and inefficient masticators.

While the present study found that a higher number of masticatory cycles reduces subjective appetite, it is not clear how they are mechanistically linked. In the present study, we measured postprandial plasma concentration of CCK and ghrelin due to their role in the regulation of appetite ${ }^{(25)}$. A higher number of masticatory cycles was associated with increased plasma CCK and reduced ghrelin, although it is not clear how mastication influenced secretion of these hormones. One possible explanation is that increasing mastication activity elicits a stronger CPR. A CPR for ghrelin and CCK has been reported by some ${ }^{(18,29)}$, but not all, studies $^{(30,31)}$. While the influence of increasing mastication on the CPR warrants further investigation, the CPR is transient and relatively small in magnitude ${ }^{(32)}$ and it is debatable whether it is sufficiently large or long lasting to influence hormone response over a period of several hours. A more likely explanation is that increasing the number of masticatory cycles before swallowing reduces the size of particles in the 

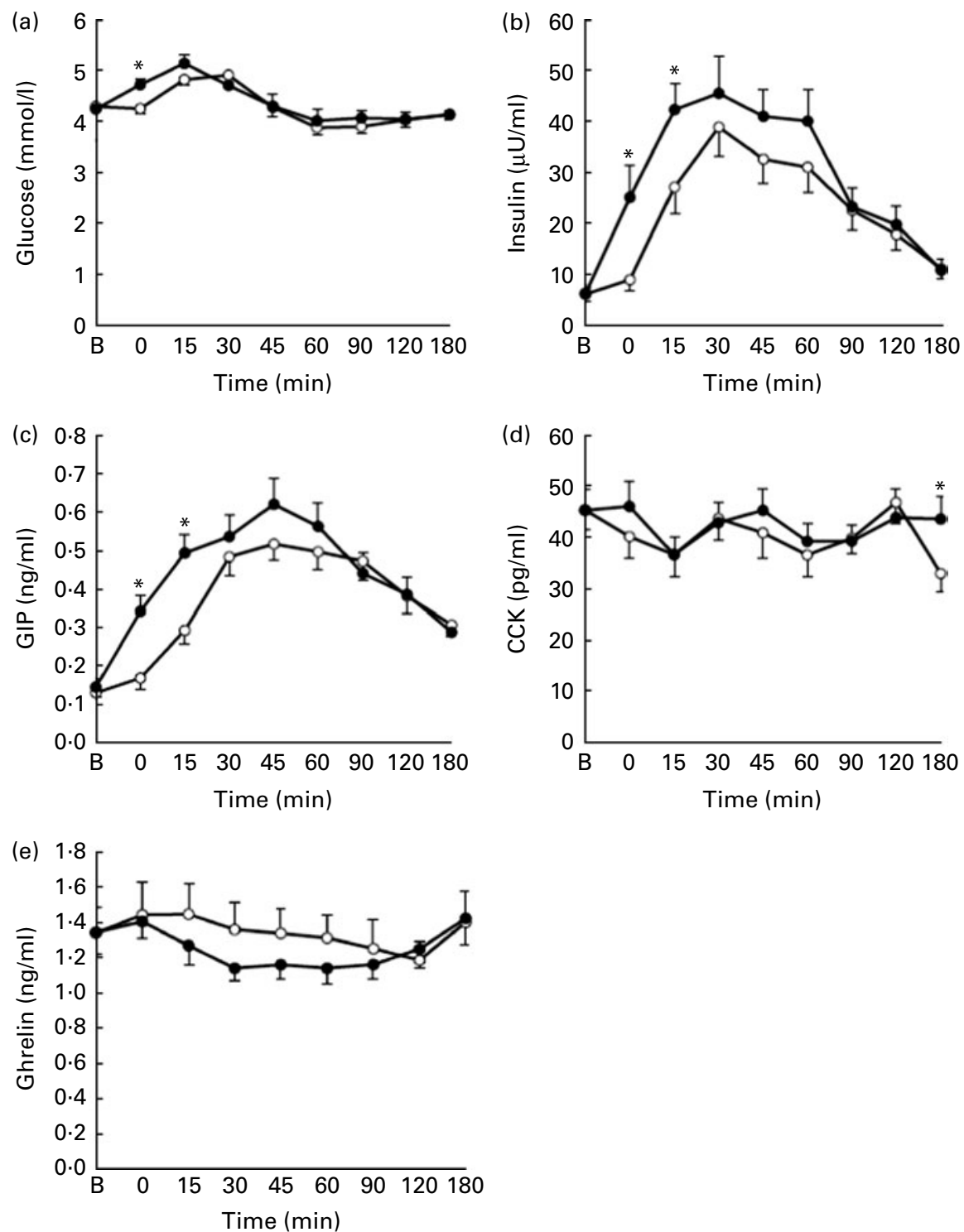

Fig. 2. Plasma level of (a) glucose, (b) insulin, (c) glucose-dependent insulinotropic peptide (GIP), (d) cholecystokinin (CCK) and (e) ghrelin following fifteen (-o-) and forty (- - ) chews conditions. Values are means, with their standard errors represented by vertical bars $(n 21)$. Mean values were significantly different for the main effect of treatment on glucose, insulin, GIP and CCK $(P=0.024, P<0.001, P<0.001$ and $P=0.045$, respectively). There was a trend towards a significant main effect of treatment on ghrelin $(P=0.051)$. ${ }^{*}$ Mean value was significantly different for plasma concentration between treatments at the same time point $(P<0.05) .1 \mu \mathrm{U}$ insulin $/ \mathrm{ml}=6.945 \mathrm{pmol}$ insulin/l.

swallowed bolus ${ }^{(33,34)}$. Reducing the size of the swallowed particles increases the bioaccessibility of nutrients due to increased breakdown of the food matrix ${ }^{(35)}$. As CCK is stimulated and ghrelin inhibited by the presence of nutrients in the gastrointestinal tract, greater nutrient bioaccessibility would presumably lead to a pattern of CCK and ghrelin secretion that promotes satiety.

While higher plasma concentrations of CCK and lower plasma ghrelin would be consistent with increased satiety, in the present study, there was no correlation between plasma concentrations of these hormones and subjective appetite, suggesting that they were not causally linked, although a lack of correlation is not uncommon in appetite studies ${ }^{(36,37)}$. Other explanations for the reduced subjective appetite may be proposed. First, several other hormones that were not measured in the present study, such as glucagon-like peptide-1 or peptide $\mathrm{YY}_{3-36}$, contribute to short-term appetite and may have had the dominant effect on appetite. Second, increasing the number of masticatory cycles before swallowing may lead to a reduction in the palatability of the pizza, which may have had a stronger effect on ratings of hunger and desire to eat during the postprandial period ${ }^{(38)}$. Third, rodent studies have shown that mastication has a direct effect on satiety centre through activation of histamine neuron $^{(14,15)}$. Further studies are required to determine through which mechanisms mastication contributes to satiety.

Food intake at a meal eaten $3 \mathrm{~h}$ after the completion of the pizza test meal was measured as a further marker of satiety. 
Despite differences in subjective appetite and plasma hormones, there was no effect of masticatory efficiency on food intake. However, in the present study, the amount eaten at the ad libitum test meal may have been influenced by factors other than appetite, masking an actual effect. First, the participants may have stopped eating before reaching satiation so that they could leave the laboratory sooner. Second, participants were not allowed to drink water with the meal. Some studies ${ }^{(39,40)}$, but not all ${ }^{(41)}$, have found that restricting fluid intake with a meal lowers food intake. It has been estimated that $75 \%$ of fluid ingestion occurs periprandially ${ }^{(42)}$, and restricting fluid intake when eating is atypical of normal behaviour for most adults. This may limit the generalisability of the data collected by the present study.

A further finding of the present study is that mastication influences the glycaemic response. Chewing the pizza forty times before swallowing resulted in a higher plasma glucose, insulin and GIP concentrations compared with chewing fifteen times. These results differ from a previous study in which it was found that chewing pork pie more times before swallowing had no effect on the plasma concentrations of glucose or insulin $^{(24)}$. This discrepancy may be due to differences in the characteristics of the test meals. This explanation is supported by a study, which found that masticatory effort influenced the glycaemic response after eating rice but not spaghetti ${ }^{(43)}$. The glycaemic response has been linked to several chronic diseases such as obesity, type 2 diabetes and heart disease ${ }^{(44)}$. Further research is required to understand the effect of variations in masticatory efficiency on the glycaemic response and disease risk.

Nonetheless, there are several limitations to the present study. A key limitation of the present study, and the previous studies using a similar experimental design ${ }^{(23,24)}$, is that the effect of mastication on appetite was not isolated from differences in eating rate, oral processing time or the physical characteristics of the swallowed bolus. Consequently, the observed effects on subjective appetite and plasma hormones cannot be solely attributed to differences in mastication and may have been due to a slower eating rate, a longer oral processing time, differences in the physical characteristics of the swallowed bolus or a combination of these factors. However, these data provide further impetus to examine the role of mastication, eating rate, oral processing time or the physical characteristics of the swallowed bolus on satiety. In addition, pizza was used as the test food, which is not typically eaten as a breakfast food. Pizza was used as it required mastication before swallowing and it provided a mix of macronutrients. Moreover, in the present study, water was not allowed throughout the test session, as gastric distention resulting from water ingestion would confound appetite measurements. While these may have resulted in an atypical meal, this effect would be consistent across both test sessions and it is unlikely that it would explain the differences in satiety or hormones between the test sessions. The study group also consisted solely of non-obese male participants to maximise the statistical power. Further studies are required to determine if different number of masticatory cycles before swallowing influences satiety in females or the obese.

\section{Acknowledgements}

We thank Visha Arumugam for her technical assistance and Jens Rehfeld for providing CCK antibody. The study was funded by Iowa State University. There are no conflicts of interest. The authors' contributions are as follows: Y. Z . and J. H. H. designed the research; Y. Z., W. H. H and J. H. H. conducted the research; Y. Z. and J. H. H. analysed the data and wrote the manuscript. All authors read and approved the final manuscript.

\section{References}

1. Lyly M, Due A, Gunnarsdottir I, et al. (2009) Foods for increasing satiety. Agro Food Ind Hi Tech 20, 42-45.

2. Apovian CM (2010) The causes, prevalence, and treatment of obesity revisited in 2009: what have we learned so far? $A m J$ Clin Nutr 91, 277S-279S.

3. Azrin NH, Kellen MJ, Brooks J, et al. (2008) Relationship between rate of eating and degree of satiation. Child Fam Behav Ther 30, 355-364.

4. Scherwitz L \& Kesten D (2005) Seven eating styles linked to overeating, overweight, and obesity. Explore (NY) 1, 342-359.

5. Andrade AM, Greene GW \& Melanson KJ (2008) Eating slowly led to decreases in energy intake within meals in healthy women. J Am Diet Assoc 108, 1186-1191.

6. Zijlstra N, de Wijk RA, Mars M, et al. (2009) Effect of bite size and oral processing time of a semisolid food on satiation. Am J Clin Nutr 90, 269-275.

7. de Wijk RA, Zijlstra N, Mars M, et al. (2008) The effects of food viscosity on bite size, bite effort and food intake. Physiol Behav 95, 527-532.

8. Zijlstra N, Mars M, de Wijk RA, et al. (2008) The effect of viscosity on ad libitum food intake. Int J Obes 32, 676-683.

9. Otsuka R, Tamakoshi K, Yatsuya H, et al. (2006) Eating fast leads to obesity: findings based on self-administered questionnaires among middle-aged Japanese men and women. J Epidemiol 16, 117-124.

10. Sasaki S, Katagiri A, Tsuji T, et al. (2003) Self-reported rate of eating correlates with body mass index in 18-y-old Japanese women. Int J Obes 27, 1405-1410.

11. Leong SL, Madden C, Gray A, et al. (2011) Faster selfreported speed of eating is related to higher body mass index in a nationwide survey of middle-aged women. $J \mathrm{Am}$ Diet Assoc 111, 1192-1197.

12. Lucas PW \& Luke DA (1986) Is food particle size a criterion for the initiation of swallowing. J Oral Rehabil 13, 127-136.

13. Frecka JM, Hollis JH \& Mattes RD (2008) Effects of appetite, BMI, food form and flavor on mastication: almonds as a test food. Eur J Clin Nutr 62, 1231-1238.

14. Sakata T, Yoshimatsu H, Masaki T, et al. (2003) Anti-obesity actions of mastication driven by histamine neurons in rats. Exp Biol Med 228, 1106-1110.

15. Sakata T, Yoshimatsu H \& Kurokawa M (1997) Hypothalamic neuronal histamine: implications of its homeostatic control of energy metabolism. Nutrition 13, 403-411.

16. Mattes RD (2000) Nutritional implications of the cephalicphase salivary response. Appetite 34, 177-183.

17. Teff KL, Mattes RD \& Engelman K (1991) Cephalic phase insulin release in normal weight males - verification and reliability. Am J Physiol 261, E430-E436.

18. Wisen O, Bjorvell H, Cantor P, et al. (1992) Plasma concentrations of regulatory peptides in obesity following modified 
sham feeding (MSF) and a liquid test meal. Regul Pept 39, 43-54.

19. Teff KL (2010) Cephalic phase pancreatic polypeptide responses to liquid and solid stimuli in humans. Physiol Behav 99, 317-323.

20. Ruijschop R, Zijlstra N, Boelrijk AEM, et al. (2011) Effects of bite size and duration of oral processing on retro-nasal aroma release - features contributing to meal termination. Br J Nutr 105, 307-315.

21. Kokkinos A, le Roux CW, Alexiadou K, et al. (2010) Eating slowly increases the postprandial response of the anorexigenic gut hormones, peptide YY and glucagon-like peptide-1. J Clin Endocrinol Metab 95, 333-337.

22. Karl J, Young A \& Montain S (2011) Eating rate during a fixed-portion meal does not affect postprandial appetite and gut peptides or energy intake during a subsequent meal. Physiol Behav 102, 524-531.

23. Cassady BA, Hollis JH, Fulford AD, et al. (2009) Mastication of almonds: effects of lipid bioaccessibility, appetite, and hormone response. Am J Clin Nutr 89, 794-800.

24. Li J, Zhang N, Hu LZ, et al. (2011) Improvement in chewing activity reduces energy intake in one meal and modulates plasma gut hormone concentrations in obese and lean young Chinese men. Am J Clin Nutr 94, 709-716.

25. de Graaf C, Blom WAM, Smeets PAM, et al. (2004) Biomarkers of satiation and satiety. Am J Clin Nutr 79, 946-961.

26. Stunkard AJ \& Messick S (1985) The 3-Factor Eating Questionnaire to measure dietary restraint, disinhibition and hunger. J Psychosom Res 29, 71-83.

27. Hsu WH, Schaffer DD \& Pineda MH (1987) Yohimbine increases plasma insulin concentrations of dogs. Proc Soc Exp Biol Med 184, 345-349.

28. Flint A, Raben A, Blundell JE, et al. (2000) Reproducibility, power and validity of visual analogue scales in assessment of appetite sensation in single test meal studies. Int $J$ Obes 24, 38-48.

29. Arosio M, Ronchi CL, Beck-Peccoz P, et al. (2004) Effects of modified sham feeding on ghrelin levels in healthy human subjects. J Clin Endocrinol Metab 89, 5101-5104.

30. Katschinski M, Dahmen G, Reinshagen M, et al. (1992) Cephalic stimulation of gastrointestinal secretory and motor responses in humans. Gastroenterology 103, 383-391.

31. Erdmann J, Lippl F \& Schusdziarra V (2003) Differential effect of protein and fat on plasma ghrelin levels in man. Regul Pept 116, 101-107.
32. Mattes RD (1997) Physiologic responses to sensory stimulation by food: nutritional implications. J Am Diet Assoc 97, 406-413.

33. Hoebler C, Devaux MF, Karinthi A, et al. (2000) Particle size of solid food after human mastication and in vitro simulation of oral breakdown. Int J Food Sci Nutr 51, 353-366.

34. Peyron MA, Mishellany A \& Woda A (2004) Particle size distribution of food boluses after mastication of six natural foods. J Dent Res $\mathbf{8 3}, 578-582$.

35. Al-Rabadi GJS, Gilbert RG \& Gidley MJ (2009) Effect of particle size on kinetics of starch digestion in milled barley and sorghum grains by porcine alpha-amylase. J Cereal Sci 50, 198-204.

36. Hagobian TA, Sharoff CG, Stephens BR, et al. (2009) Effect of exercise on energy-regulating hormones and appetite in men and women. Am J Physiol Regul Integr Comp Physiol 296, R233-R242.

37. MacIntosh CG, Andrews JM, Jones KL, et al. (1999) Effect of age on concentrations of plasma cholecystokinin, glucagon-like peptide-1, and peptide YY and their relation to appetite and pyloric motility. Am J Clin Nutr 69, 999-1006.

38. Sorensen LB, Moller P, Flint A, et al. (2003) Effect of sensory perception of foods on appetite and food intake: a review of studies on humans. Int J Obes 27, 1152-1166.

39. Russell K \& Delahunty C (2004) The effect of viscosity and volume on pleasantness and satiating power of rice milk. Food Qual Prefer 15, 743-750.

40. Rolls BJ, Bell EA \& Thorwart ML (1999) Water incorporated into a food but not served with a food decreases energy intake in lean women. Am J Clin Nutr 70, $448-455$.

41. Triana AJ, Apanius V, Richmond C, et al. (2003) Restricting fluid intake during a single meal did not affect food intake in older adults. Appetite 41, 79-86.

42. McKiernan F, Houchins JA \& Mattes RD (2008) Relationships between human thirst, hunger, drinking, and feeding. Physiol Behav 94, 700-708.

43. Ranawana V, Henry CJK \& Pratt M (2010) Degree of habitual mastication seems to contribute to interindividual variations in the glycemic response to rice but not to spaghetti. Nutr Res 30, 382-391.

44. Augustin LS, Franceschi S, Jenkins DJA, et al. (2002) Glycemic index in chronic disease: a review. Eur J Clin Nutr 56, 1049-1071. 